Recepción: 25 / 10 / 2016

Aceptación: 18 / 01 / 2017

Publicación: 29 / 04 / 2017

Ciencias de la salud

Artículo de investigación

\title{
Diabetes mellitus. Factores de riesgo en los adultos mayores
}

\author{
Mellitus diabetes. Risk Factors in the Elderly
}

\section{Diabetes mellitus. Fatores de risco em adultos mais velhos}

\author{
Martha A. Ayala-Paredes ${ }^{\mathrm{I}}$ \\ aliciadevillagrán@ hotmail.com \\ Víctor H. Rivera-García "I \\ dr_vhriverag@hotmail.com \\ Cecil H. Flores-Balseca III \\ cecil.floresb@ug.edu.ec
}

Correspondencia: aliciadevillagrán@ hotmail.com

- Magister en Gerencia Educativa, Especialista en Gestión de Procesos Educativos, Diplomado en Docencia Superior, Psicóloga Industrial, Docente de la Universidad de Guayaquil, Guayaquil, Ecuador.

" Especialista en Oftalmología, Doctor en Medicina y Cirugía, Docente de la Universidad Estatal de Guayaquil, Guayaquil, Ecuador, Docente de la Universidad de Guayaquil, Guayaquil, Ecuador.

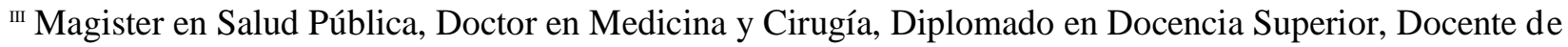
la Universidad de Guayaquil, Guayaquil, Ecuador. 



\section{Resumen}

Se realizó un estudio descriptivo transversal, de 162 pacientes mayores de 60 años con diabetes mellitus, en la ciudad de Guayaquil, en el periodo con vistas a describir los factores de riesgo asociados a la diabetes mellitus. La muestra quedó constituida por 60 pacientes, seleccionados mediante un muestreo no probabilístico al azar, que cumplieron los criterios de inclusión siguientes: tener 60 años y más, haber dado su consentimiento para participar en la investigación y no presentar enfermedad invalidante que afectara su capacidad intelectual y/o física. Entre las variables seleccionadas figuraron: edad, sexo, enfermedades asociadas, hábitos tóxicos, práctica de ejercicios, dieta. Los resultados mostraron un predominio del sexo femenino en pacientes diabéticos, la hipertensión arterial fue la asociada con mayor prevalencia, en la muestra de estudio asociada e insuficiente práctica de ejercicios físicos, quienes no cumplían con la dieta establecida y alto consumo de café y fumadores.

Palabras clave: adulto mayor; diabetes mellitus; factores de riesgo.

\section{Abstract}

A descriptive cross-sectional study of 162 patients over 60 years of age with diabetes mellitus was conducted in the city of Guayaquil in the period to describe the risk factors associated with diabetes mellitus. The sample consisted of 60 patients, selected by random non-probabilistic sampling, who met the following inclusion criteria: being 60 years and over, having given their consent to participate in the research and not presenting invalidating disease that would affect their intellectual capacity And / or physical. Among the selected variables were: age, sex, associated diseases, toxic habits, exercise practice, diet. The results showed a predominance of the female sex in diabetic patients, hypertension was associated with higher prevalence, in the associated study sample and insufficient physical exercise practice, who did not comply with the established diet and high consumption of coffee and smokers.

Keywords: elderly; mellitus diabetes; risk factor's.

\section{Resumo}

Um estudo descritivo transversal de 162 pacientes com mais de 60 anos de idade com a diabetes mellitus, na cidade de Guayaquil, no período, a fim de descrever os factores de risco associados com a diabetes mellitus foi realizada. A amostra foi composta de 60 pacientes, selecionados por 
meio de uma amostragem não probabilística aleatória, que preencheram os seguintes critérios de inclusão: ter 60 anos e mais, ter dado o seu consentimento para participar da pesquisa e não têm doença debilitante que afeta sua capacidade intelectual e / ou física. Entre as variáveis escolhidas eles incluíram: idade, sexo, doenças associadas, hábitos tóxicos, exercícios práticos, dieta. Os resultados mostraram uma predominância do sexo feminino em pacientes diabéticos, hipertensão foi associada com uma maior prevalência na amostra do estudo associado e exercício físico insuficiente, que não preenchia estabelecida e alto consumo de café e fumar dieta.

Palavras chave: idosos; diabetes mellitus; fatores de risco.

\section{Introducción}

Alrededor de un $20 \%$ de adultos mayores son portadores de diabetes mellitus y casi un $40 \%$ tienen intolerancia a la glucosa. El tipo de diabetes más frecuentemente presentada por el adulto mayor, es la diabetes tipo 2 con un alto componente de insulina, resistencia acentuada por el proceso de envejecimiento y con una insulina deficiencia relativa; la diabetes tipo 1 de origen autoinmune, que lleva a la destrucción total de las células beta del páncreas, ha sido descrita en un porcentaje bastante bajo en adultos mayores. ${ }^{1}$

La palabra diabetes data del siglo II, cuando Areteo de Capadocia la denomina con este nombre. Willis en el siglo XVII describe el sabor dulce (azúcar, miel) como la miel de la orina en la diabetes y le da el atributo de mellitus. ${ }^{2}$

Esta enfermedad crónica aparece en el adulto mayor a causa de la disminución de la secreción de insulina, asociada al envejecimiento y por una mayor resistencia a la insulina, debido a un incremento en la proporción de grasa corporal y disminución de la masa muscular. Sin embargo la mayor cantidad de diabéticos adultos mayores, vienen arrastrando su enfermedad desde que eran adultos más jóvenes. Con los años se producen cambios sustanciales en la función de las células beta y en la acción de la insulina, aumento del tejido adiposo visceral, aumento de la resistencia a la acción de la insulina, ácidos grasos libres y péptidos producidos en el tejido graso (adiponectina, TNF- alfa, leptina), reducción de la masa muscular esquelética e infiltración de grasa en el tejido muscular, altera la captación de glucosa. ${ }^{3}$

El cuidado de un adulto mayor con diabetes, puede implicar enfrentar retos especiales como: la coexistencia de condiciones médicas, limitaciones físicas y falla de memoria. 
Algunos de los factores únicos en el cuidado de un adulto mayor con diabetes incluyen:

- Asegurarse de que la persona reciba una nutrición adecuada.

- Administrar los medicamentos de forma personal, para cerciorarte que los toma apropiadamente y prevenir efectos secundarios por la interacción de medicamentos.

- Lidiar con limitaciones físicas o mentales que pueden hacer difícil el cuidado de la diabetes.

Una de las principales dificultades en la atención al paciente diabético, continúa siendo la falta de educación hacia un estilo de vida y una actitud favorable hacia la enfermedad, lo cual solo puede lograrse mediante estrategias educativas; para ello deben involucrarse los 3 niveles del sistema nacional de salud, especialmente la atención primaria. La DM, es una afección compleja que puede afectar todos los aspectos de la vida diaria de la persona que la padece y el mejor de los tratamientos falla, si el enfermo no participa día a día en el control de sus niveles de glucemia. ${ }^{4}$

Un informe reciente de la Organización Mundial de la Salud (OMS), apunta de forma convincente la asociación entre aumento de peso y obesidad, resaltando que el consumo alimentario habitual, el sedentarismo y la DM 2, constituyen factores esenciales relacionados con el desarrollo de enfermedades crónicas no transmisibles. Asimismo, la reducción de factores de riesgo modificables como el peso, consumo alimentario y la práctica de actividad física mostró un potencial significativo en la reducción del riesgo para padecer la diabetes sacarina, inclusive en individuos con historia familiar de esta enfermedad. ${ }^{5}$

La educación de las personas que presentan esta afección, es un componente esencial de las estrategias de prevención y tratamiento, aunque no reemplaza el tratamiento médico, pero proporciona el estímulo necesario para encarar un cambio radical en el estilo de vida, las nuevas concepciones de la educación y la promoción del derecho a la educación para la salud, si se quiere garantizar la participación activa en el control y tratamiento eficaz de la diabetes sacarina. ${ }^{6}$ La diabetes mellitus es en sí misma una causa de morbilidad, discapacidad o muerte y a la vez un factor de riesgo de otras enfermedades, por lo que constituye un problema creciente de salud, tanto en el mundo desarrollado como subdesarrollado. Lo anterior se convierte en motivos para avanzar en el conocimiento de esta situación, que se ha convertido en un problema de salud pública afectando la calidad de vida de la población. ${ }^{7}$ 


\section{Materiales y métodos}

Se realizó un estudio descriptivo transversal de 162 pacientes mayores de 60 años con diabetes mellitus, pertenecientes a la ciudad de Guayaquil, en el periodo con vistas a describir los factores de riesgo asociados a la diabetes mellitus.

La muestra quedó constituida por 60 pacientes, seleccionados mediante un muestreo no probabilístico al azar, que cumplieron los criterios de inclusión siguientes: tener 60 años y más, haber dado su consentimiento para participar en la investigación y no presentar enfermedad invalidante, que afectará su capacidad intelectual y/o física.

El criterio de salida que se tomó en cuenta fue que abandonaran por cualquier causa el estudio.

Entre las variables seleccionadas figuraron:

- Edad: variable cuantitativa continúa, se registró en años cumplidos y se agrupó según los siguientes intervalos: 60-69, 70-79, 80-89, 90 años y más.

- Sexo: variable cualitativa nominal dicotómica, según sus dos variables biológicas (masculino y femenino).

- Enfermedades asociadas: hipertensión arterial, dislipidemias, cardiopatía isquémica, polineuropatía periférica y desnutrición.

- Hábitos tóxicos:

- Café: tres o más tomas en el día o su equivalente en una toma (aproximado de tres tazas pequeñas).

- Hábito de fumar: se consideraron aquellos que referían la práctica del mismo o su antecedente independientemente del tiempo de duración, la cantidad, el tipo y modo de consumo.

- Ingestión de bebidas alcohólicas: se tuvieron en cuenta los que ingerían cualquier tipo de estas.

- Cumplimiento de los pilares del diabético: dieta, práctica de ejercicios físicos y tratamiento.

En el caso de la dieta se tuvo en cuenta los siguientes criterios:

- Se consideró que sí cumplían con la misma cuando manifestaron ingerir de 4 a 6 comidas. 
- No cumplían cuando solo hacían las tres comidas (desayuno, almuerzo y comida) o menos de estas, además si se alimentaban cada vez que querían.

- Práctica de ejercicios físicos: se recogieron las respuestas según conceptos argumentados a continuación:

- Práctica correcta de ejercicios físicos: pacientes incorporados a la actividad física, de manera regular al menos tres veces por semana y de poca intensidad (caminatas, ejercicios aeróbicos, labores agrícolas ligeras).

- Práctica incorrecta de ejercicios físicos: aquellos que realizaban con una periodicidad inferior a tres veces por semanas o de una intensidad que requería gran esfuerzo físico como: subir escaleras, largas caminatas, levantamiento de pesos.

- No práctica de ejercicios: los que de forma esporádica realizaban alguna que otra actividad física independientemente de su intensidad.

- En cuanto al tratamiento medicamentoso, se consideró si su cumplimiento fue regular o irregular y se operacionalizó de la siguiente manera:

- Regular: cuando manifestaron que siempre cumplían el tratamiento con medicamentos.

- Irregular: cuando cumplían el tratamiento con medicamentos solo en ocasiones, casi nunca o nunca.

\section{Resultados}

Del total de pacientes con diabetes mellitus (tabla 1), el sexo femenino estuvo representado con 66,6\% y el masculino aportó 33.3\%. El grupo de edad de 60-69, fue el de mayor predominio de dicha afección con $51.6 \%$, de ellos 22 eran mujeres y 9 hombres.

Tabla 1. Ancianos diabéticos según edad y sexo

\begin{tabular}{|c|c|c|c|c|c|c|}
\hline \multirow{2}{*}{$\begin{array}{c}\text { Grupo de } \\
\text { edades }\end{array}$} & \multicolumn{4}{|c|}{ Sexo } & \multicolumn{2}{|c|}{ Total } \\
\cline { 2 - 7 } & \multicolumn{2}{|c|}{ Masculino } & \multicolumn{2}{c|}{ Femenino } & \multicolumn{2}{|c|}{} \\
\cline { 2 - 7 } & No & $\%$ & No & $\%$ & No & $\%$ \\
\hline $60-69$ & 9 & 45.0 & 22 & 55.0 & 31 & 51.6 \\
\hline
\end{tabular}




\begin{tabular}{|c|c|c|c|c|c|c|}
\hline $70-79$ & 7 & 35.0 & 10 & 25.0 & 17 & 28.3 \\
\hline $80-89$ & 3 & 15.0 & 5 & 12.5 & 8 & 13.3 \\
\hline+90 & 1 & 5.0 & 3 & 7.5 & 4 & 6.6 \\
\hline Total & $\mathbf{2 0}$ & $\mathbf{3 3 . 3}$ & $\mathbf{4 0}$ & $\mathbf{6 6 . 6}$ & $\mathbf{6 0}$ & $\mathbf{1 0 0}$ \\
\hline
\end{tabular}

Como se muestra en la tabla 2, al determinar las enfermedades asociadas que padecían estos gerontes, se alcanzó como resultado un predominio de la hipertensión arterial (37,5\% y 35,0\% respectivamente), con el sexo femenino como el más significativo; las mismas estuvieron seguidas por la cardiopatía isquémica en 11 pacientes del sexo femenino (27.5\%) y 9 del sexo masculino (45.0\%). Cabe destacar la existencia de 6 pacientes con desnutrición, el cual represento el $10.0 \%$ del total de senescentes.

Tabla 2. Ancianos diabéticos según sexo y enfermedades asociadas

\begin{tabular}{|l|c|c|c|c|c|c|}
\hline \multirow{2}{*}{$\begin{array}{l}\text { Enfermedades } \\
\text { asociadas }\end{array}$} & \multicolumn{5}{|c|}{ Sexo } & \multicolumn{2}{c|}{ Total } \\
\cline { 2 - 7 } & Masculino & \multicolumn{2}{c|}{ Femenino } & \multicolumn{2}{c|}{} \\
\cline { 2 - 7 } & No & $\%$ & No & $\%$ & No & $\%$ \\
\hline $\begin{array}{l}\text { Hipertensión } \\
\text { arterial }\end{array}$ & 7 & 35.0 & 15 & 37.5 & 22 & 36.6 \\
\hline Dislipidemia & 2 & 10.0 & 5 & 12.5 & 7 & 11.6 \\
\hline $\begin{array}{l}\text { Cardiopatía } \\
\text { isquémica }\end{array}$ & 9 & 45.0 & 11 & 27.5 & 20 & 33.3 \\
\hline $\begin{array}{l}\text { Polineuropatía } \\
\text { periférica }\end{array}$ & 1 & 5.0 & 4 & 10.0 & 5 & 8.3 \\
\hline Desnutrición & 1 & 5.0 & 5 & 12.5 & 6 & 10 \\
\hline Total & $\mathbf{2 0}$ & $\mathbf{3 3 . 3}$ & $\mathbf{4 0}$ & $\mathbf{6 6 . 6}$ & $\mathbf{6 0}$ & $\mathbf{1 0 0}$ \\
\hline
\end{tabular}

Obsérvese en la tabla 3, un predominio de los gerontes que no practicaban ejercicios ni desarrollaban actividades, que pudieran contribuir a realizar algún tipo de actividad física, representados por $70.0 \%$ de la muestra estudiada. Resulta importante destacar que existió un alza 
de las féminas con este factor (30 para 75,0\%); además, no se encontraron ancianos con práctica incorrecta de ejercicios físicos.

Tabla 3. Ancianos diabéticos según sexo y práctica de ejercicios físicos

\begin{tabular}{|l|c|c|c|c|c|c|}
\hline \multirow{2}{*}{$\begin{array}{l}\text { Realización de } \\
\text { ejercicios } \\
\text { físicos }\end{array}$} & \multicolumn{2}{|c|}{ Masculino } & \multicolumn{2}{c|}{ Femenino } & \multicolumn{2}{c|}{ Total } \\
\cline { 2 - 7 } & No & $\%$ & No & $\%$ & No & $\%$ \\
\hline $\begin{array}{l}\text { Practica de } \\
\text { ejercicios } \\
\text { físicos }\end{array}$ & 8 & 40.0 & 10 & 25.0 & 18 & 30.0 \\
\hline $\begin{array}{l}\text { No practica de } \\
\text { ejercicios } \\
\text { físicos }\end{array}$ & 12 & 60.0 & 30 & 75.0 & 42 & 70.0 \\
\hline Total & $\mathbf{2 0}$ & $\mathbf{3 3 . 3}$ & $\mathbf{4 0}$ & $\mathbf{6 6 . 6}$ & $\mathbf{6 0}$ & $\mathbf{1 0 0}$ \\
\hline
\end{tabular}

En cuanto a los hábitos tóxicos presentes en estos ancianos, resultó que 92,0\% de ellos ingerían café, donde hubo una representación femenina. En su totalidad, el porcentaje de adultos mayores fumadores y los que ingerían bebidas alcohólicas fue similar 30,0\% y 28,0\% respectivamente.

El cumplimiento de la dieta por parte de los enfermos con diabetes mellitus, evidenció que 28 $(56,9 \%)$ solo cumplían con tres comidas al día, o se alimentaban cada vez que querían, el resto de la muestra estudiada no suplían sus necesidades dietéticas.

Al determinar el cumplimiento del tratamiento medicamentoso, se constató que 48 pacientes cumplían con el mismo de forma regular $(80.0 \%)$ y solo 12 lo consumían irregularmente.

Al indagar por la percepción de su estado de salud, los adultos mayores con diabetes mellitus, manifestaron en un 57,7\% $(n=35)$ que su condición de salud es excelente o muy bueno, en tanto el 41,6\% (n=25) refirió todo lo contrario.

\section{Discusión}

El envejecimiento poblacional, es uno de los factores de riesgo determinantes en la frecuencia de la DM2, en los últimos años. Los resultados obtenidos (tabla 1) se asemejan a estudios realizados 
por diferentes autores en Panamá $^{8}$, los cuales muestran que la población femenina en este grupo de edades, es la más afectada por la DM. (84.4\%). En otro estudio realizado por Sarabia Alcocer ${ }^{9}$ donde el sexo femenino predomino con un 55.0\%.

Se considera que demográficamente existen más féminas que hombres en una proporción de 1:7, esta puede ser una de las causas de estos resultados; además, en las mujeres después de los 50 años suceden cambios hormonales, que favorecen la obesidad y el sedentarismo, cambian su estilo de vida y presentan malos hábitos dietéticos que pueden estar arraigados desde su juventud, factores que pueden contribuir a padecer dicha enfermedad. ${ }^{2}$

El conocimiento de los factores de riesgo específicos en la comunidad, permitirá desarrollar actividades preventivas, promotoras, y políticas de salud a fin de modificarlos en la población de mayor riesgo y de ese modo disminuir las tasas de la enfermedad y de sus complicaciones.

Autores camagüeyanos coinciden con los resultados evidenciados en la tabla 2, al encontrar la hipertensión arterial (HTA) como la dolencia que predominó en su investigación. ${ }^{10}$

Estudios realizados por Framigham, abordan que el riesgo para la aparición de un evento cardiovascular, es mayor en un paciente con HTA y que además es diabético, que en uno que no presente esta enfermedad. ${ }^{11}$

La elevada prevalencia de hipertensión arterial y su asociación con otros factores de riesgo cardiovasculares como diabetes, obesidad y dislipidemia, apuntan la necesidad de realizar intervenciones específicas de enfermería e implementar un protocolo de atención, que tenga como enfoque minimizar las complicaciones provenientes de la hipertensión, como también prevenir el surgimiento de otras enfermedades cardiovasculares. ${ }^{12}$

Entre las ECVs, la hipertensión arterial sistémica (HAS), constituye un importante factor de riesgo para el surgimiento de complicaciones cardíacas y cerebro vasculares ${ }^{13}$, siendo considerada un problema de salud pública en el ámbito mundial. En 2000 la prevalencia de la HAS, en la población mundial era de 25\% y la estimativa para el año de 2025 es de 29\% (2). En estudios realizados, en Brasil la prevalencia de la hipertensión varió entre 22,3\% y 43,9\%, con promedio de $32,5 \% .^{14,15}$ 
Resulta importante determinar las enfermedades asociadas a la DM, pues las políticas de la intervención educativa están en dependencia de ello y de otros factores que determinan en muchas ocasiones complicaciones sobreañadidas.

La inactividad física es un importante factor de riesgo para muchas condiciones y problemas crónicos de salud, tales como las enfermedades cardiovasculares, la hipertensión, la obesidad, la osteoporosis, la diabetes mellitus y de salud mental. ${ }^{16}$

En los Estados Unidos, el 60\% de las personas consideradas mayores, no participan regularmente en actividades físicas ${ }^{8-11}$, y en otros países desarrollados, entre el $30-80 \%$ de las personas son físicamente inactivas. ${ }^{16}$

El ejercicio regular ha sido indicado para mejorar el control de la glucosa sanguínea, reducir los factores de riesgo cardiovascular, contribuir a la pérdida de peso, aumentar la sensibilidad a la insulina y proporcionar una sensación de bienestar general. Además, el ejercicio regular puede impedir la aparición de la DM tipo 2 en personas con riesgo de padecerla. ${ }^{17}$

Los efectos beneficiosos de la actividad física sobre la sensibilidad a la insulina, parecen ser el resultado final de la suma de los efectos específicos, sobre el contenido de los receptores GLUT 4 , capacidad oxidativa y densidad capilar del músculo esquelético. ${ }^{16}$ Datos preliminares sugieren que el transporte de glucosa independiente de la insulina inducidos por el ejercicio, está promovido por el aumento de la producción endotelial y muscular de óxido nítrico [ON]. ${ }^{17,18}$

Por lo tanto, el ejercicio físico puede mejorar los niveles de ON y también la disfunción endotelial observada en las personas con DM. ${ }^{17}$

Un estudio realizado en Güines, demuestra que la mayoría de los pacientes estudiados no practicaban ejercicios físicos ${ }^{18}$, lo cual coincide con los resultados de la tabla 3.

El tratamiento médico nutricional, es un componente esencial del cuidado y el tratamiento global del paciente diabético. Su enfoque va más allá del control glucémico y se extiende a la HbA1c, el control del peso, los valores lipídicos y la presión arterial (PA). ${ }^{19}$

La modificación de los patrones alimentarios no es tarea fácil. El objetivo final implica que el paciente se sienta cómodo y seguro, con un plan alimentario personalizado que incorpore 
alimentos que sean de su agrado y que mantenga los patrones dietéticos propios, en la medida de lo posible, evitando las comidas entre horas. ${ }^{19}$

Los resultados de esta serie coinciden con los obtenidos por Domínguez y otros, ${ }^{4}$ al reflejar en su investigación que los pacientes no consumían la dieta adecuadamente y que era difícil asimilarla por poseer patrones incorrectos. Asimismo, un estudio realizado en Río de Janeiro, mostró que los afectados no cumplían con el tratamiento dietético. ${ }^{20}$ Se debe comprender, que más que indicar una dieta estricta, la cual con frecuencia el enfermo no realiza, es necesario señalar normas dietéticas comprensibles y con posibilidades de ser cumplidas, cercanas al patrón alimentario habitual, compatibles con sus ingresos económicos y otras limitaciones.

La investigación realizada por Salazar Martínez y otros autores, sugiere una asociación inversa importante entre el consumo de café y el riesgo de diabetes tipo 2 en hombres y mujeres, el café contiene cantidades importantes de potasio, niacina, magnesio y sustancias antioxidantes, de efectos favorables en el desarrollo de la diabetes mellitus. ${ }^{21}$ Dichos investigadores consideran que estos resultados, se deben al arraigo popular de estos hábitos nocivos, puesto que toda la población tiene acceso a los cigarros, tabacos, alcohol y café por expedirse de forma liberada en todos los comercios.

En un estudio realizado en México, se encontró que la mayoría de los pacientes diabéticos no cumplían con el tratamiento, debido a las malas condiciones socioeconómicas, puesto que esta es una enfermedad que requiere atención médica y una importante demanda de medicamentos. Estos resultados no concuerdan con los descritos en este trabajo. ${ }^{22}$

\section{Conclusiones}

Los resultados mostraron un predominio del sexo femenino en pacientes diabéticos, la hipertensión arterial fue la enfermedad asociada con mayor prevalencia en la muestra de estudio asociada e insuficiente práctica de ejercicios físicos, quienes no cumplían con la dieta establecida y alto consumo de café y fumadores.

\section{Referencias bibliográficas}

1- Santiesteban S S. Diabetes mellitus en el adulto mayor, 2003. [citado 8 Ago 2016]. 42(4) Disponible en: http://www.fihu-diagnostico.org.pe/revista/numeros/2003/julago03/165-169.html 
2- De la Paz Castillo K, I Proenza Fernández, I. Gallardo Sánchez, I Y, Fernández Pérez S y Mompié Lastre A. Factores de riesgo en adultos mayores con diabetes mellitus. Revista medisan 2013 [citado 12 ago 2016]; 16(4): 489 Disponible en: http://bvs.sld.cu/revistas/san/vol_16_4_12/san01412.htm

3- Garibay O. Diabetes Mellitus en el adulto mayor. 2014. [citado 12 julio 2016] Disponible en: https://es.slideshare.net/OswaldoAngeles/diabetes-mellitus-en-el-adulto-mayor

4- Domínguez Bofill S, Cortina Mena I, Bello Rodríguez B. Intervención educativa en pacientes diabéticos en la parroquia Valle de Tucutunemo. Rev méd electrón. 2008 [citado 14 junio 2016]; 30(2). Disponible en: http://www.revmatanzas.sld.cu/revista\%20medica/ano\%202008/vol2\%202008/tema06.htm

5- Carolino IDR, Molena-Fernándes CA, Tasca RS, Marcon SS, Cuman RKN. Factores de riesgo en pacientes con diabetes mellitus tipo 2. Rev Latino-am Enfermagem. 2008; 16(2):238-44

6- Pérez Delgado A, Alonso Carbonell L, García Milián AJ, Garrote Rodríguez I, González Pérez S, Morales Rigau JM. Intervención educativa en diabéticos tipo 2. Rev Cubana Med Gen. 2009 [citado 14 mayo 2016]; 25(4). Disponible en: http://scielo.sld.cu/scielo.php?script=sci_arttext\&pid=S0864-

$21252009000400003 \& \operatorname{lng}=$ pt\&nrm=iso\&tlng=es

7- Suarez Flórez L E, Chavarriaga Ríos M C. Prevalencia de diabetes mellitus autoreportada y factores asociados en los adultos de 60-64 años de Medellín y área metropolitana, participantes en la encuesta nacional de demografía y salud y la encuesta de situación nutricional en Colombia del año 2010. CES Salud Pública. 2014 [citado 14 mayo 2016]; 5: 21-37. Disponible en: https://dialnet.unirioja.es/descarga/articulo/4804769.pdf

8- Regla Carolino I R Molena-Fernándes CA, Tasca RS, Marcon SS, Cuman RKN. Factores de riesgo en pacientes con diabetes mellitus tipo 2. Rev Latino-am Enfermagem 2008 [citado 14 mayo 2016]; 16(2) Disponible en: www.scielo.br/pdf/rlae/v16n2/es_11.pdf

9- Sarabia Alcocer B, Can Valle C, Guerrero Ceh J. Identificación de Factores de Riesgo de la Diabetes Mellitus Tipo 2 en Adultos de 30 a 60 Años de edad en la Comunidad de Isla Aguada, 
Municipio de Ciudad del Carmen, Campeche. Revista iberoamericana para la investigación y el desarrollo educativo. 2015 [citado 14 mayo 2016]; 5(10)

10- Ramos Cabrera A, Aguilar Rodríguez MC, Victoria Bárzaga HO, Manresa Martínez I. Estudio comparativo de la incidencia de diabetes mellitus en dos áreas de salud. AMC. 2008 [citado 14 junio 2016]; 12(2). Disponible http://scielo.sld.cu/scielo.php?script=sci_arttext\&pid=S1025-02552008000200007\&lng=es

11- Vasan R, Beiser A, Seshadri S, Larson MG, Kannel WB, D’Agostino RB, et al., Residual lifetime risk for developing hipertension in middle-aged women and men. The Framingham Heart Study. JAMA. 2002; 287(8):1003-10

12- Trindade Radovanovic C A, Afonso dos Santos L, Dalva de Barros Carvalho4 M, Silva Marcon S. Hipertensión arterial y otros factores de riesgo asociados a las enfermedades cardiovasculares en adultos. Rev. Latino-Am. Enfermagem 2014; 22(4):547-53

13- World Health Organization (WHO). Global Atlas on Cardiovascular Disease Prevention and Control. Mendis S, Puska P, Norrving B editors. Geneva: World Health Organization; 2011

14- Cesarino CB, Cipullo JP, Martin JFV, Ciorlia LA, Godoy MRP, Cordeiro JA, et al., Prevalência e fatores sociodemográficos em hipertensos de São José do Rio Preto. Arq Bras Cardiol. 2008; 91(1):31-5

15- Rosário TM, Scala LCNS, França GVA, Pereira MRG, Jardim PCBV. Prevalência, controle e tratamento da hipertensão arterial sistêmica em Nobres, MT. Arq Bras Cardiol. 2009; 93(6):6728.

16- Landinez Parra N, Contreras Valencia C, Castro Villamil. Proceso de envejecimiento, ejercicio y fisioterapia. Revista cubana de salud pública 2012; 38(4): 562-580

17- Hernández Rodríguez J; Licea Puig M E. Papel del ejercicio físico en las personas con diabetes mellitus. 2010. [citado 14 mayo 2016]. Disponible en: http://www.bvs.sld.cu/revistas/end/vol21_2_10/end06210.htm

18- Díaz Díaz O, Valenciaga Rodríguez JL, Domínguez Alonso E. Comportamiento epidemiológico de la diabetes mellitus en el municipio de Güines: Año 2002. Rev. Cubana 
Higiene y Epid. 2004 [citado 12 Sept 2016]; 42 (1). Disponible en: http://scielo.sld.cu/scielo.php?script=sci_arttext\&pid=S1561$30032004000100003 \& \operatorname{lng}=\mathrm{es} \& n r m=i s o \& t \operatorname{lng}=e s$

19- González Sarmiento E, Pascual Calleja I, Laclaustra Gimeno M, J Casasnovas Lenguas. Síndrome metabólico y diabetes mellitus. Rev Esp Cardiol Supl. 2005 [citado 12 Sept 2016]; 5(D):30-7. Disponible en: http://www.revespcardiol.org/es/sindrome-metabolico-diabetesmellitus/articulo/13083446/

20- Pérez Delgado A, Alonso Carbonell L, García Milián AJ, Garrote Rodríguez I, González Pérez S, Morales Rigau JM. Intervención educativa en diabéticos tipo 2. Rev Cubana Med Gen Integr. 2009 [citado 14 mayo 2016]; 25 (4). Disponible en: http://scielo.sld.cu/scielo.php?script=sci_arttext\&pid=S0864$21252009000400003 \& \operatorname{lng}=$ pt\&nrm=iso\&tlng=es

21- Salazar-Martinez E, Willett WC, Ascherio A, Manson JE Leitzmann MF, Stampfer MJ, Hu FB. Coffee consumption and risk for type 2 diabetes mellitus. Ann Intern Med. 2004; 140:1-8.

22- Vázquez Castellanos JL, Panduro Cerda A. Diabetes mellitus tipo 2: Un problema epidemiológico y de emergencia en México. Investigación en Salud [Internet]. 2001 [citado 10 junio 2016]; 3:18-21. Disponible en: http://redalyc.uaemex.mx/pdf/142/14239904.pdf 